\title{
An effective transmit packet coding with trust-based relay nodes in VANETs
}

\author{
Omar A. Hammood ${ }^{1}$, Mohd Nizam Mohmad Kahar², Waleed A. Hammood ${ }^{3}$, Raed A Hasan ${ }^{4}$, \\ Mostafa Abdulghfoor Mohammed ${ }^{5}$, Ayoob A. Yoob ${ }^{6}$, Tole Sutikno ${ }^{7}$ \\ ${ }^{1,2,3,4}$ Department of Computer System and Software Engineering, University Malaysia Pahang, Malay sia \\ ${ }^{5}$ Faculty of Automatic Control and Computers, University Polytechnic of Bucharest, Romania \\ ${ }^{6}$ School of Communication and Information Engineering, Huazhong University for Science and Technology, China \\ ${ }^{7}$ Department of Electrical Engineering, Faculty of Industrial Technology, Universitas Ahmad Dahlan, Indonesia
}

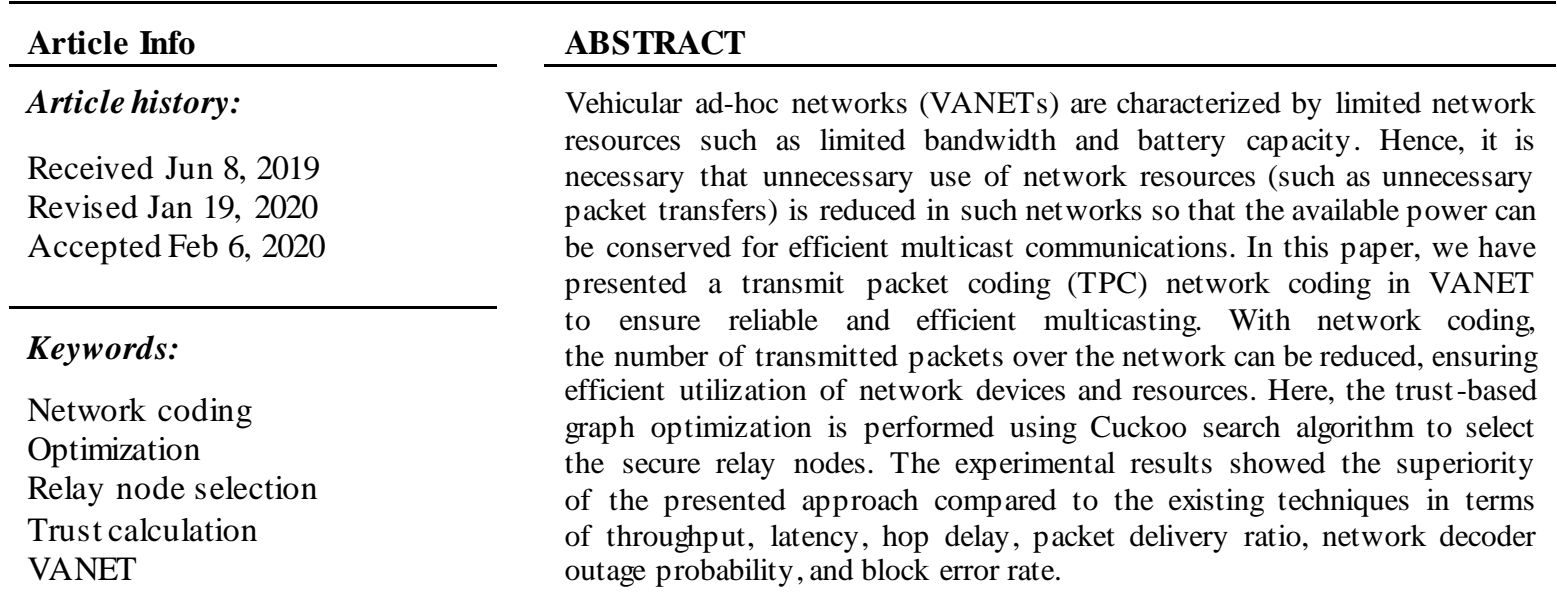

This is an open access article under the $\underline{C C B Y-S A}$ license.

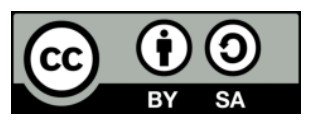

\section{Corresponding Author:}

Omar A. Hammood,

Department of Computer System and Software Engineering,

University Malaysia Pahang (UMP),

Kuantan-26300, Pahang, Malaysia.

Email: omer_almajeed@yahoo.com

\section{INTRODUCTION}

In recent years, researchers have imagined that data communication can be made progressive and proficient. We send information as packets, which are mixed in a totally arbitrary manner toward one end, and descrambled at the opposite end in a communication network, rather than sending plain information. Network coding was acquainted with the idea to upgrade the through put of the systems; however, at this point, it is utilized to expand the capability, robustness, tomography, and security of systems [1, 2]. Network coding is characterized as applying codes at nodes in a system. It is a technique for sending messages over the network and for restoring lost information [3]. Network coding in a network is used to encode data acquired from the input links. It has been being used in various executions of information interchange networks, like the Internet, peer-to-peer networks, wireless sensor networks (WSNs), shared systems, cognitive radio networks (CRNs), multicast systems, VANETs, and other rising networks [4].

As of late, VANETs have picked up great fame among industry and scholarly research networks. VANETs use vehicles as versatile hubs in an ad-hoc manner. Each contributing vehicle acts as a remote switch or hub to interconnect with one another. The fixed street side units like traffic light towers set up 
a backbone system to provide network, and in time conveyance, dependable and reliable security-related data. The VANETs standards and executions help in developing quantities of remote items that would now be able to be utilized in vehicles [5-7]. VANET is an ad-hoc mobile system that uses vehicles on the streets as network devices as they form a distributed communication network for message exchanges. A wide scope of utilization can be beneficial for such innovation. Safety applications are road-side security measures that are in place to ensure the safety of road users [8-10].

VANETs are perfect to keep up a high class of administration in strong technological topologies, giving quick steering and security. These challenges affect the testing and utilization of new systems (such as network coding (NC) and cooperative correspondences) to enhance the quality of overall communication experience [11-13]. For this situation, we can accomplish better data transmission use, lessen the system crashes, and diminish the quantity of rebroadcasting occasions by utilizing strategies, for example, the network coding. It is a realized strategy to effectively use data transmission by abusing the communication idea of the remote medium. Network coding diminishes the number of retransmissions by enabling the relay nodes to advance the data pack and to do a type of logic task on them [14-16].

Numerous researchers have proposed distinct network coding methods in VANETs to improve multi-hop routing procedures. In [17-19], a protocol is proposed which uses powerfully produced back-bone vehicles to disperse data pack in the network. Moreover, they utilized network coding to improve data pack gathering likelihood. Another protocol utilizes intra flow and inter-flow coding [18, 19] to decrease the protocol overhead when contrasted with existing protocols. In [20-22] a robust and fast forwarding (ROFF) protocol was proposed for the reduction of excess retransmissions in the system. In [23-25], a multihop communication protocol was presented for time-basic emergency messages (EMs) dispersal. This proposed trinary apportioned black-burst-based communicate protocol (3P3B) applies trinary packing at the system layer and mini-distributed interframe space (DIFS) device at the medium access control (MAC) sublayer. Celimuge et al. [26-28] proposed fuzzy broadcast (FUZZBR), a fuzzy logic based multi-hop communicate protocol which has a low message overhead because of its utilization of just specific tran sfer hubs for data distribution [29-32]. At the point when a transfer hub neglects to retransmit a data pack, the FUZZBR additionally utilizes a lightweight retransmission mechanism [33-35].

The main contributions of this paper are as follows: 1) adaptive quantum logic gate based (AQLG) network coding is presented in VANET in order to achieve efficient and reliable multicasting; and 2) trust-based graph optimization is performed using Cuckoo search algorithm for the effective selection of trustable relay nodes. The structure of the manuscript is composed as Section 2 surveys the papers regarding the proposed strategy. In sections 3, a brief discussion about the proposed approach is presented; section 4 investigates the exploratory outcomes, and section 5 concludes the paper.

\section{RELATED WORK}

A cross-layer design for both amplify-and-forward (AF) and decode-and-forward two-way relaying (DF-TWR) was proposed by Yanping et al. [21] based on the NC method for the enhancement of the achievable throughput under explicit quality of service (QoS) requirements, such as the most reasonable error rate and delay. They naturally amalgamate adaptive analog network coding (ANC) and network coded modulation (NCM) with truncated automatic repeat request (ARQ) worked at the distinctive OSI layers. Several improved NC-based ARQ procedures were intended at the data-link layer based on the Stop-andWait and the Selective Repeat ARQ techniques. However, adaptive ANC/NCM was summoned at the physical layer based on the estimated packet error ratio (PER). They built up a novel transmission procedure for choosing the ideal group sizes for the relay to the destination connections which must be adjusted to both pair of channel conditions.

A cross-layer analysis structure and execution assessments of cooperative ARQ with opportunistic multi-point relaying (OMPR) in versatile systems were proposed by Hsin-Li \& Sau-Hsuan [22]. This proposal considered 3 exemplar OMPR functions and their combinations for opportunistic distributed space-time coded (ODSTC) retransmissions in time-division duplex LTE-A frameworks. As evidenced by the coordination delays of the OMPR functions, it is possible to adequately assess the influence of channel blurring on the spatial and temporal varieties as sociated with ODSTC-based ARQ, as well as their effects on the transmission energy consumption, framework throughput, and relays queuing delays under the system. According to the analytical outcomes, a rating technique was additionally proposed to augment the framework throughput subject to requirements on packet error rates at mobile stations (MSs) and queuing delays at relay stations (RSs).

Arghyadip \& Rajarshi [23] proposed consistent communication in a real-life topology, where connection misfortune probabilities were unique in relation to one another. They determined the closed-form expressions and examined as ymptotic conducts for the expected number of transmissions using stop-and-hold 
up ARQ and NC as error control components. In the physical layer cooperative broadcast, numerous hubs can transmit all things considered to exploit spatial variety. They integrate the possibility of physical layer cooperative communication with $\mathrm{ARQ}$ and $\mathrm{NC}$ independently, to acquire further unwavering quality. The numerical outcomes based on their asymptotic examination showed that NC offers more consistency than stop-and-hold up ARQ. In any case, as the size of the participating set increases, both plans offer similar dependability.

Lingzhi Li et al. [24] proposed a network coding with crowdsourcing-based trajectory estimation (NC/CTE) method for data transmission in VANET. Key points were pre-designated in the movement area. At a different time, every node estimates the key points discovered by the other nodes in the explored area using a crowdsourcing method that depends on GPS pre-trajectory navigation. NC, recoding and reverse forwarding are employed for data propagation based on the outcome of the trajectory estimation by the nodes.

Angelos et al. [25] proposed a cross-layer systematic model for the investigation of network coding based ARQ medium access control (MAC) protocols in correlated moderate blurred conditions, where two end hubs are supported by a group of relays to trade information packages. Angelos et al. [25] presented an NC-based MAC protocol for VANETs which depends on the cooperative ARQ techniques. This protocol controls channel access between a set of relays and can use NC to reduce the total number of transmissions, thus improving the networks' performance in terms of QoS metrics.

The existing works have shown that link interruption can occur due to is sues like the inter-vehicular mobility links, excessive inter-vehicular spacing, and message transmission failures. Being that only one relay is involved in the communication, the effects of the collision and idles lots on the network's performance can be ignored. Moreover, increased energy consumption, and decreased transmission security are the problems [21-25] in the existing works. The existing works contributions and their benefits and limitations are given in Table 1.

Table 1. The existing works

\begin{tabular}{|c|c|c|c|}
\hline Author & Main contribution & Benefits & Limitations \\
\hline Yanping et al. [21] & Truncated-ARQ & $\begin{array}{l}\text { - Bidirectional network coding } \\
\text { scenarios. } \\
\text { - Higher Throughput }\end{array}$ & $\begin{array}{l}- \text { Need to extend the } \\
\text { physical layer techniques. } \\
\text { - Improvement is needed } \\
\text { in Latency }\end{array}$ \\
\hline $\begin{array}{l}\text { Hsin-Li \& Sau-Hsuan } \\
\text { [22] }\end{array}$ & $\begin{array}{l}\text { ARQ with OMPR in } \\
\text { mobile net works. }\end{array}$ & $\begin{array}{l}\text { - Provides fewer energy } \\
\text { consumptions } \\
\text { - Provides near best system } \\
\text { throughput. }\end{array}$ & - Delay is not reduced. \\
\hline $\begin{array}{l}\text { Arghyadip Roy and } \\
\text { Rajarshi Roy [23] }\end{array}$ & $\begin{array}{l}\text { NC over stop-and-wait } \\
\text { ARQ in a practical access } \\
\text { point model. }\end{array}$ & $\begin{array}{l}\text { - Proposed network coding offers } \\
\text { more reliability }\end{array}$ & $\begin{array}{l}\text { - Go-back-N and selective } \\
\text { repeat ARQ with NC. }\end{array}$ \\
\hline Lingzhi Li et al. [24] & NC/CTE & $\begin{array}{l}\text { - It improves the reliability and } \\
\text { scalability of vehicular networks. }\end{array}$ & $\begin{array}{l}\text { - Need to change the } \\
\text { coding steps in NC/CTE } \\
\text { for less overhead ratio in } \\
\text { the fut ure. }\end{array}$ \\
\hline $\begin{array}{l}\text { Angelos Antonopoulos } \\
\text { et al. [25] }\end{array}$ & $\begin{array}{l}\text { NC-based ARQ MAC } \\
\text { protocols }\end{array}$ & $\begin{array}{l}\text { - Energy efficiency, high } \\
\text { throughput, and highlighting the } \\
\text { importance of cross-layer } \\
\text { approaches. }\end{array}$ & $\begin{array}{l}\text { - Low enhancement of the } \\
\text { current NC-based MAC } \\
\text { protocols in terms of } \\
\text { performance. }\end{array}$ \\
\hline
\end{tabular}

The objectives of this work are: i) to give general physical (PHY) layer theoretic expressions for assessing vital system parameters, pertinent in two-way broadcast, ii) to show how these expressions are combined in hypothetical models of the upper layers (i.e., MAC), and iii) to examine the execution of newly proposed NC- assisted cooperative ARQ (NCCARQ) MAC procedure under interrelated shadowing conditions.

\section{PROPOSED METHODOLOGY}

TPC-MAC was developed for the arrangement of the transmissions between a set of relay nodes in a bidirectional manner between 2 hubs in vehicular networks. Principally, TPC-MAC was developed to empower the versatile stations in requesting for connectivity via a set of trusted nodes upon receiving an incorrect information package. Secondly, the proposed protocol was developed to enable the relay nodes to execute $\mathrm{NC}$ on packets before their transmission. Figure 1 represents a simple scenario of the basic concept of the proposed TPC-MAC. The scenario in Figure 1 considered wireless communication between 
a base station (S) and a mobile destination (D). In this situation, many packets will be errone ously received since the destination vehicle is moving along the boundaries of the source's range. However, vehicles moving within the transmission range (between source and destination) constitute potential relays due to the high chance of correctly receiving the propagated data.

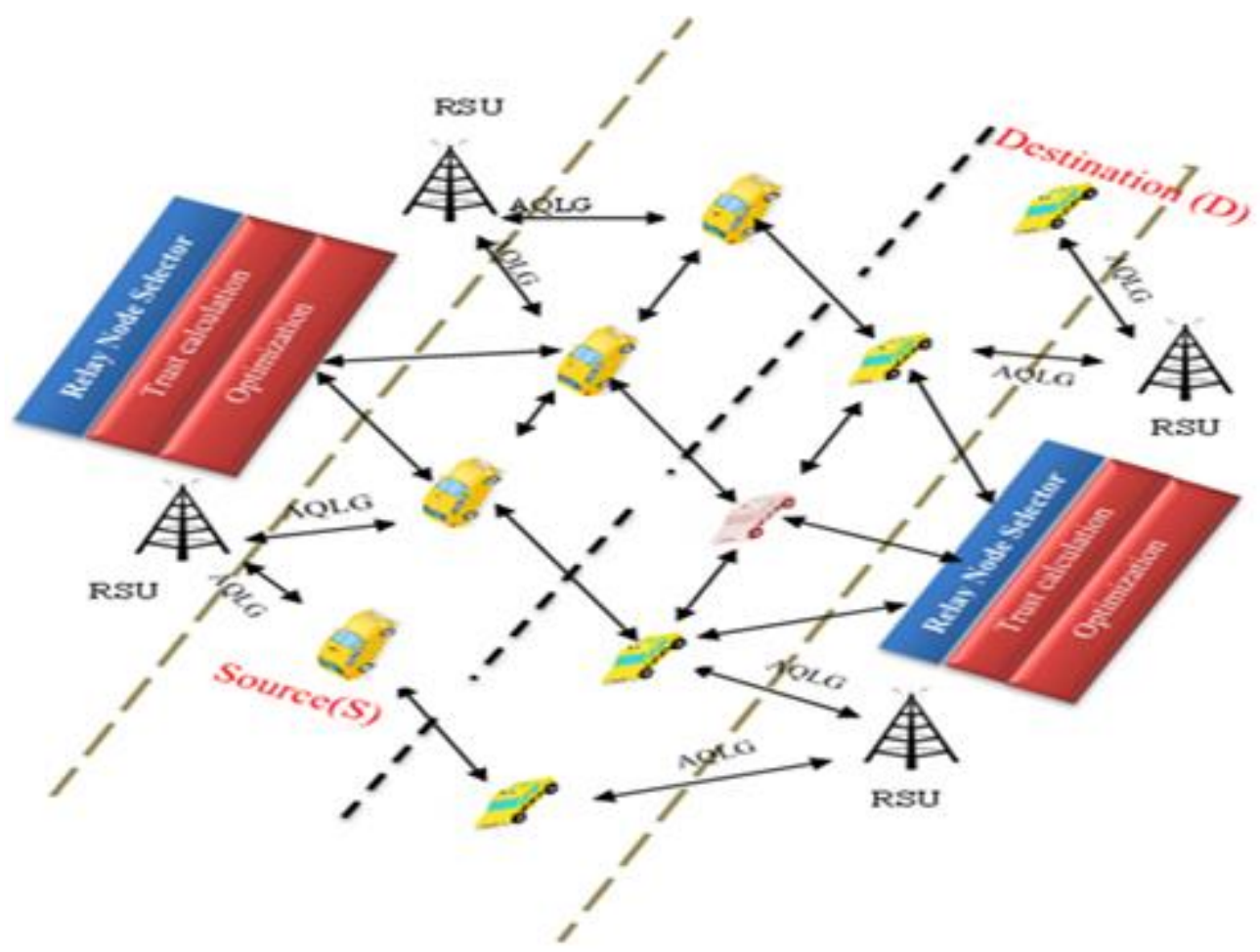

Figure 1. General idea of proposed TPC operation

The VANET comprises a set of nodes $\bar{N}=\left\{n_{1}, n_{2}, n_{3} \ldots \ldots n_{k}\right\}$ and assumes the source as $n_{1}$ and the destination as $n_{k}$. In the proposed work, data is transferred from the source to the destination nodes through the relay nodes. To avoid failures in data transmission, the reliable relay nodes are selected by utilizing trust-based graph optimization. The trust graph optimization is performed by utilizing the Cuckoo search algorithm adapted to the topology of the cooperation cluster relay nodes selection. The detailed explanation of the proposed design process is discussed in the subsequent sections.

\subsection{Trust calculation for relay nodes}

Trust is the level of consistency about the data. Trust can similarly be portrayed as the confidence level of a specific node to complete allotted work within some time. The trust of hubs is calculated by the condition (1).

$$
T_{n}=\frac{\sum_{i=1}^{n} D_{i} * \tau}{\sum_{i=1}^{n} D_{i}}
$$

where, $T_{r}$ is the trust of the node, $\tau$ is the level of discrete trust, $D_{i}$ is determined by the condition (2);

$$
D_{i}=\Pi\left(1-\alpha_{t}\right)
$$

where $T_{k}$ is the trust of every task of node and $\alpha_{t}$ is determined by the condition (3);

$$
\alpha_{t}=\frac{1}{1+t}
$$

Here, $t$ is the task processing time of a specific node. At that time, the cuckoo search optimization algorithm selecting the relay nodes viably dependent on the determined trust esteems. 


\subsection{The behavior of the relay selection}

As per [26], the relay ensures the maintenance of a queuing buffer in a bid to keep the data received from the slower source; meanwhile, if the source is sending data in the same rate, the relay is chosen randomly. In our proposed technique, the optimized relay is selected using Cuckoo search algorithm recently presented by Yang and Deb. To apply this as an optimization tool, Yang and Deb utilized three ideal rules:

a. Every cuckoo lay one egg, which signifies a set of solution coordinates, at a time and dumps it in an arbitrary nest;

b. A portion of the nests comprising the best eggs, or solutions, will extend to the next subsequent generation;

c. The quantity of nests is fixed and there is a likelihood that a host can find an outsider egg. If this occurs, the host can either dispose of the egg or the nest and this outcome in creating another nest in a new location.

Based on these idealized rules, the basic steps of the CS can be summarized as shown in the pseudocode presented in Algorithm 1.

Algorithm 1: Pseudocode of the Cuckoo Search (CS)

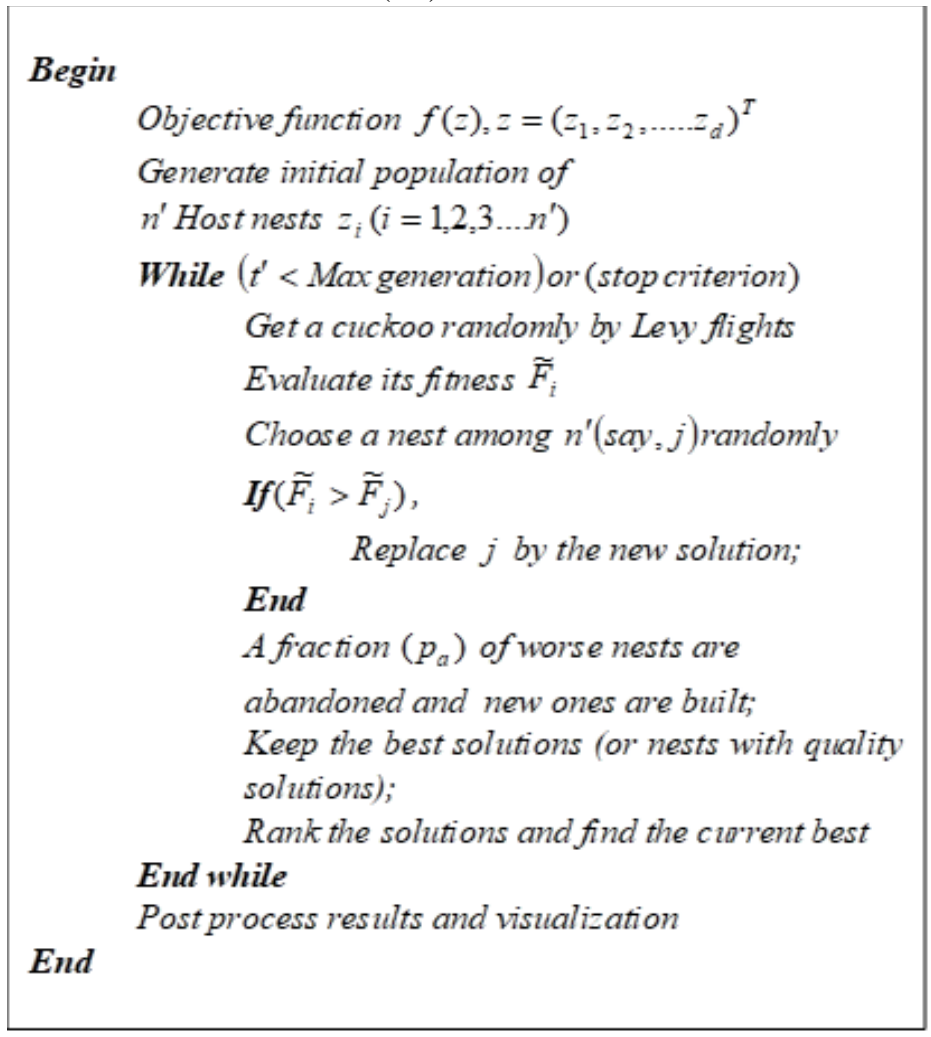

For each iteration $\left(t^{\prime}\right)$, a cuckoo egg $i$, is chosen arbitrarily and different solutions $z^{\left(t^{\prime}+1\right)}$ are produced. This arbitrary search accomplished more effectively by utilizing Levy flights rather than with a straightforward arbitrary walk. The Levy flights are a sort of arbitrary walk in which the steps are characterized in terms of the step-lengths that pursue specific likelihood dissemination in which the directions of the steps must be isotropic and arbitrary. For this situation, the general condition for the Levy flight is specified by,

$$
z_{i}^{\left(t^{\prime}+1\right)}=z_{i}^{\left(t^{\prime}\right)}+\alpha \oplus \operatorname{Levy}(\beta)
$$

where the superscript $t^{\prime}$ is utilized to demonstrate the current generation, the symbol $\bigoplus$ is utilized to designate the entry-wise multiplication and $\alpha>0$ represents the step size. This step size decides how far a particle can move by arbitrary walk for a fixed number of cycles. The transition probability of the Levy flights in condition (5) is modulated by the Levy dissemination as:

$$
\operatorname{Levy}(\beta) \sim g^{-\beta},(1<\beta \leq 3)
$$


The condition (5) has an infinite change with an infinite mean. From the execution viewpoint, primarily, a random direction regarding to uniform distribution is preferred; then, the sequence of steps succeeding the selected Levy distribution is produced. The cuckoo search optimization algorithm assesses the fitness of the new solution and relates it with the present one. At that point, the current solutions are ordered at every iteration step dependent of their fitness and the finest solution is reached so far is kept as the vector $\left(z_{\text {best }}^{\prime}\right)$. The algorithm is applied iteratively until a prescribed stopping criterion is met.

\section{Node Insertion}

For node insertion $z_{\text {best }}^{\prime}$ esteems are considered which the trust results of every node in VANET. Each node is then categorized according to their trust values. At last, the secure nodes are chosen as relay nodes dependent on the trust values for the esteems for the information transmission.

\section{Link insertion}

These chosen nodes are then connected to other nodes which are not a part of source and destination. The node which holds the higher trust esteem is chosen as reliable nodes for the information transmission. By means of the reliable nodes data is transmitted from the source to the destination without failures.

\subsection{Transmit packet coding}

The proposed work in this study aims at achieving a better bandwidth utilization, as well as minimize network congestion during multimedia data transmission. Therefore, the NC technique was used to minimize the number of data retransmissions. Our proposed work environment is similar to the given Figure 1, where, the source is positioned at the opposite terminals of the same road, sharing $\mathrm{N}$ hop's area of interest. The non-safety data are disseminated by the source to the vehicles in the shared road section between them.

Here, instead of having the relay broadcasting, our proposed work utilizes the trust-based optimized relay selection for the data transmission and encoding into one packet prior to their rebroadcasting in the network. This minimizes the bandwidth utilization and delays at the relay. It is assumed that the relay can forward the packets and execute AQLG operation on the transmitted packets prior to their rebroadcasting. It is also believed that the relay is aware of the average data rates sent by the source.

\subsubsection{TPC operational example}

Simple network topology with four stations is considered, every one of them in the transmission range of one another. A source node $\left(n_{1}\right)$ transmits a data packet $\left(A^{\prime}\right)$ to a destination node $\left(n_{k}\right)$ that does likewise have a packet $\left(B^{\prime}\right)$ intended to the source station. Besides, there are two helper or relay nodes $\left(\bar{H}_{1}\right.$ and $\left.\bar{H}_{2}\right)$ that support this specific bidirectional communication. The complete process is described as pursues,

Instant 1: At instant $t_{1}^{\prime}$, source node $n_{1}$ transmits the data packet $A^{\prime}$ to destination node $n_{2}$.

Instant 2: Upon reception, at instant $t_{2}^{\prime}$, destination node $n_{2}$ neglects to demodulate the information packet, therefore broadcasting a packet request $(P R)$ packet requesting for the cooperation of the neighboring relay nodes along with the data packet $B^{\prime}$, destined to the source node $n_{1}$.

Instant 3: The acceptance of $P R$ at instant $t_{3}^{\prime}$ activates the nodes $\bar{H}_{1}$ and $\bar{H}_{2}$ to become activated relays and check the trust based optimized set up their link to participate in the dispute contention phase.

Instant 4: At instant $t_{4}^{\prime}$, the setup link of $\bar{H}_{1}$ terminates and $\bar{H}_{1}$ transfers the coded packet $A^{\prime} B^{\prime}$ to the nodes $n_{1}$ and $n_{k}$ concurrently.

Instant 5: At instant $t_{5}^{\prime}$, the destination node $n_{k}$ retrieves the original packet $A^{\prime}$ and sends back an acknowledgment $(A C K)$ packet subsequently it can decipher legitimately the Adaptive quantum logic based coded packet.

Instant 6: At instant $t_{6}^{\prime}$, the source node $n_{1}$ acknowledges the packet $B^{\prime}$ since it can decode appropriately the coded packet $A^{\prime} B^{\prime}$. The network topology operation with transmit packet coding is depicted in Figure 2.

The proposed AQLG-MAC protocol is intended to organize the transmissions among a group of relays that encourages a bidirectional inter-nodal communication in VANET. The first necessity of AQLG-MAC is that all nodes in the system ought to work in indiscriminate so as to have the ability to tune in to all on-going transmissions and cooperate whenever required. The second fundamental necessity of AQLG-MAC is that a duplicate of all the received packet must be stored by the nodes until an acknowledgment is received from the destination station. The stations that receive the $P R$ packet becomes the potential candidates that will serve as the active relays for communication. Accordingly, the formation 
of the relay set is initiated after gathering the $P R$, while the participant's stations prepare to propagate their information. Being that the packets designated to the destination and source have been stored by the partners, they utilize the transmit packet coding technique to produce a new coded packet. Consequently, the active relays strive to access the channel in a bid to transmit the TPC packet.

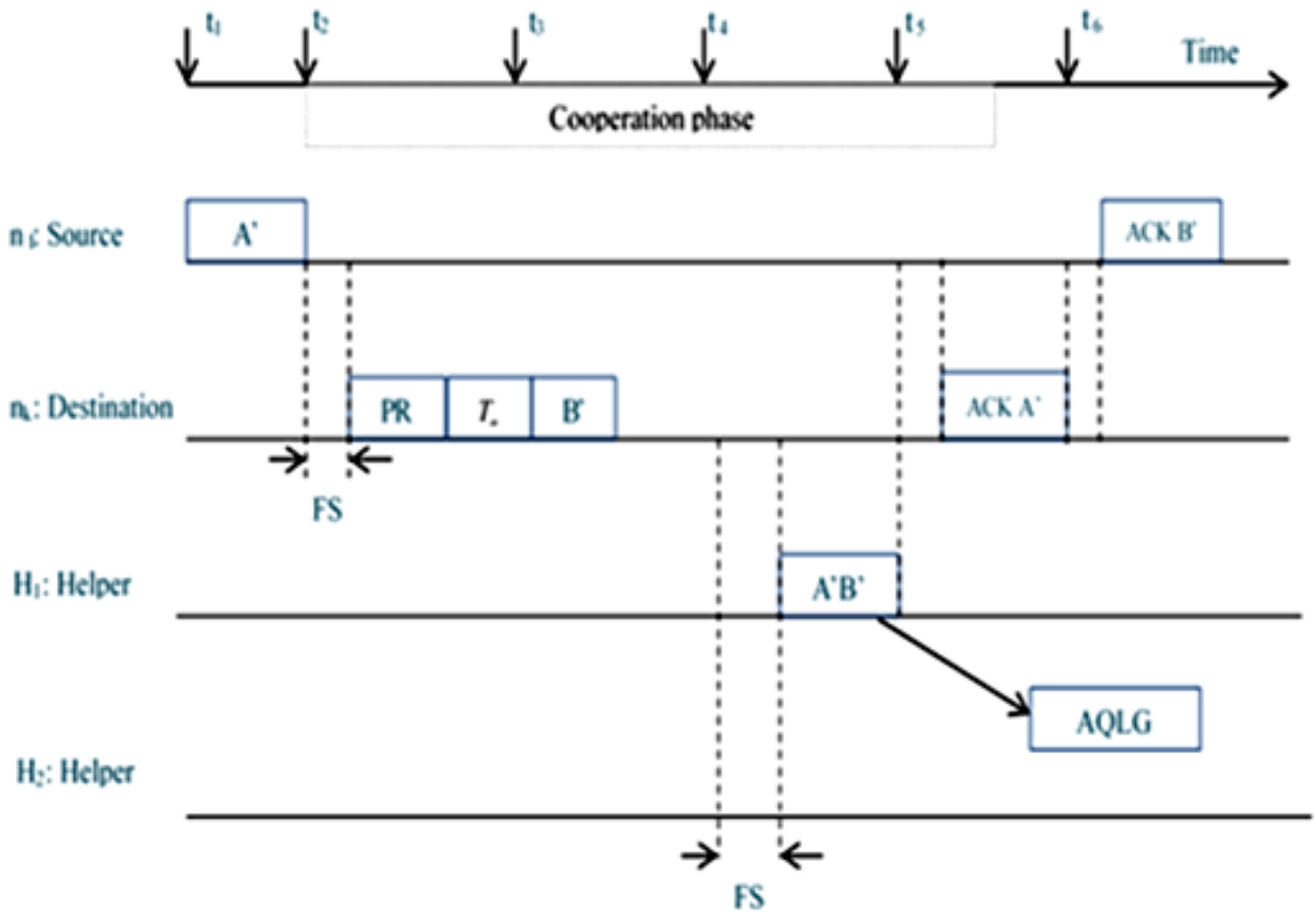

Figure 2. Network topology operation with a frame sequence

\subsubsection{Adapti ve quantum logic coding}

Quantum coding is a state which is not equal to straightforwardly produce of subsystems; this is additionally clarified mathematically. For example, there are two relay nodes $R_{A} R_{B}$ and the corresponding composited framework $R_{A B}$. The quantum conditions of subsystems as well as composited system

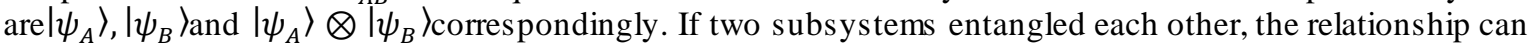
be described as pursues,

$$
R_{A B}=R_{A} \otimes R_{B}
$$

Nevertheless,

$$
\left|\psi_{A B}\right\rangle \neq\left|\psi_{A}\right\rangle \otimes\left|\psi_{B}\right\rangle
$$

There is a composited quantum bit $\left|\psi_{A B}^{+}\right\rangle$, which is quantum entangled state, furthermore, $\left|\psi_{A}\right\rangle$ and $\left|\psi_{B}\right\rangle$ is entangled pair. It must fulfill the beneath condition,

$$
\left|\psi_{A B}^{+}\right\rangle=\frac{1}{\sqrt{2}}\left\{\left|0_{A}\right\rangle \otimes\left|1_{B}\right\rangle+\left|1_{A}\right\rangle \otimes\left|0_{B}\right\rangle\right\}
$$

The feature of this condition can be described that when the, $\left|\psi_{A}\right\rangle$ is $|0\rangle$ the state of $\left|\psi_{B}\right\rangle$ certainly is opposite $|1\rangle$ vice versa. However, when $\left|\psi_{A}\right\rangle$ is collapsed to Eigen state $|1\rangle$ by measurement, $\left|\psi_{B}\right\rangle$ unavoidably collapses to opposite Eigen state $|0\rangle$ vice versa. Likewise, the data packet is coded before the transmission to secure the transmission data. Finally, the coded data packet is transmitted through the chosen relay nodes to the destination node securely. The overall flowchart of the proposed technique is given in Figure 3.

Once the network coded packet has been received by the source and the destination from the relay, they can decode the packet and retrieve the respective original data packets. Later, they will acknowledge the receipt of the packet by sending the respective ACK, thereby, ending the cooperation phase. Upon 
receiving the ACK, the relays are informed about the completion of specific communication, so they can delete the packets of their buffers. Should the received coded packets fail to be retrieved after a certain maximum cooperation timeout as a result of transmission errors, the relays will be mandated to re-forward the NC packet.

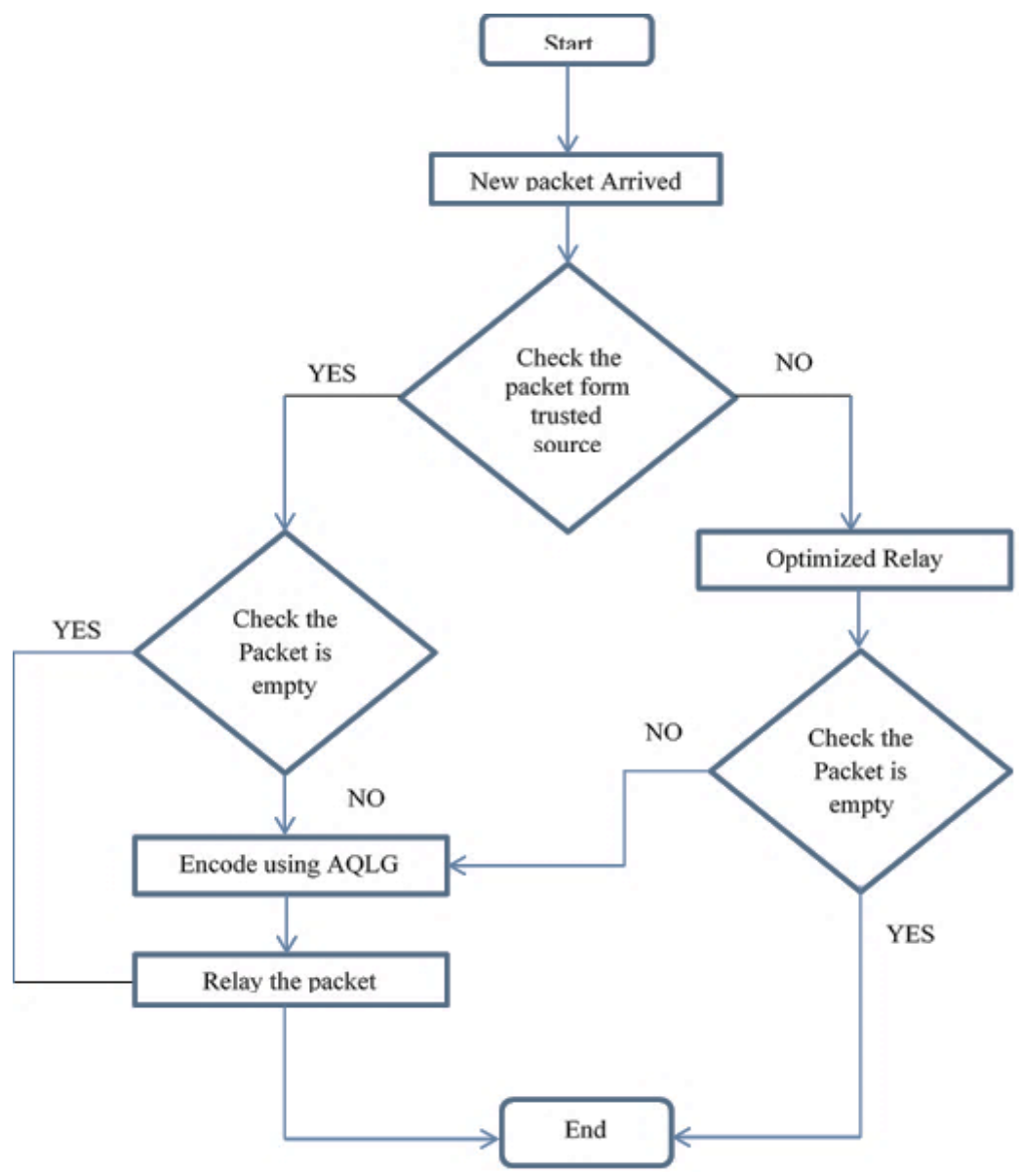

Figure 3. Flow chart of the proposed network coding

\section{RESULTS AND DISCUSSION}

The implementation of our proposed adaptive quantum logic-based network coding is performed in the working platform of MATLAB. In order to examine the performance of the proposed transmit packet, coding is distinguished with the existing random linear coding (RLC) [27], NCCARQ-MAC [28] protocol and Huffman coding [26] techniques in terms of throughput, hop delay, network decoder outage probability, block error rate, latency, and packet delivery ratio. Table 2 presents the parametric configuration of the network based on the IEEE 802.11 physical layer. The time for NC application to the data packe ts was considered insignificant since the coding takes place between only two packets.

\begin{tabular}{cc}
\multicolumn{2}{c}{ Table 2. System parameters } \\
\hline Parameter & Value \\
\hline MACheader & 64 bytes \\
PHY header & $96 \mu \mathrm{s}$ \\
ACK, RFC & 14 bytes \\
DAT A packets & 1000 bytes \\
SIFS & $10 \mu \mathrm{s}$ \\
DIFS & $20 \mu \mathrm{s}$ \\
\hline
\end{tabular}

Moreover, this study considered different network scenarios with different SNR values between the source and the destination. The transmission of the control packets was executed at the rate of $6 \mathrm{Mb} / \mathrm{s}$, 
while the data packets were transmitted at the rate of 6,28 and $64 \mathrm{Mb} / \mathrm{s}$ for low, medium and high SNR values, respectively. With the positioning of the relays close to the destination, it was assumed that in all the scenarios, the transmission rates are 6 for control packet and $64 \mathrm{Mb} / \mathrm{s}$ for the data packets. The 3 unique simulation scenarios are presented in Table 3.

Table 3. Simulation scenarios

\begin{tabular}{ccccc}
\hline SNR(S-D) & $\begin{array}{c}\text { Source control } \\
\text { rate }(\mathrm{Mb} / \mathrm{s})\end{array}$ & $\begin{array}{c}\text { Source data rate } \\
(\mathrm{Mb} / \mathrm{s})\end{array}$ & $\begin{array}{c}\text { Relay control rate } \\
(\mathrm{Mb} / \mathrm{s})\end{array}$ & $\begin{array}{c}\text { Relay data rate } \\
(\mathrm{Mb} / \mathrm{s})\end{array}$ \\
\hline Low & 6 & 6 & 6 & 64 \\
Medium & 6 & 28 & 6 & 64 \\
High & 6 & 64 & 6 & 64 \\
\hline
\end{tabular}

The performance of the proposed TPC is compared with the existing random linear coding (RLC), NCCARQ-MAC, and Huffman coding techniques regarding throughput, latency, hop delay, packet delivery ratio (PDR), block error rate (BER), and network decoder outage probability. The comparison results in terms of different performance measures are given in Table 4.

Table 4. Comparis on of performance of proposed coding with existing RLC and Huffman coding

\begin{tabular}{ccccccc}
\hline Methods & Throughput & Latency & Hop delay & PDR & BER & Outage probability \\
\hline Proposed AQLGcoding & $5.42 \times 10^{8}$ & $1.32 \times 10^{-6}$ & $9.72 \times 10^{-5}$ & 92.81 & 0.10 & 0.81 \\
Random Linear Coding & $4.53 \times 10^{8}$ & $1.45 \times 10^{-6}$ & 0.010 & 90.62 & 0.15 & 0.975 \\
Huffman coding & $4.09 \times 10^{8}$ & $1.59 \times 10^{-0}$ & 0.012 & 88.42 & 0.16 & 0.987 \\
NCCARQ-MAC & $3.37 \times 10^{8}$ & $1.52 \times 10^{-6}$ & 0.012247 & 87.22555 & 0.205701 & 1 \\
\hline
\end{tabular}

\subsection{Throughput}

Throughput is the maximum amount of data which can be effectively transmitted within one scheduled time. A systems' total throughput is the sum of the overall throughput created by the direct and effective transmissions of bits plus the throughput determined by the cooperation phase after receiving the erroneous packet. Mathematically, the throughput of a systemis represented as:

$$
\begin{aligned}
& \bar{S}_{\text {total }}=\bar{S}_{D}+\bar{S}_{\text {co-operative }} \\
& \bar{S}_{D}=\left(1-\overline{P E R_{S \rightarrow D}}\right) * \frac{\bar{P}_{\text {load }}}{\bar{T}_{D}} \\
& \bar{S}_{\text {co-operative }}=2 * \overline{P E R_{S \rightarrow D}} * \frac{\bar{P}_{\text {load }}}{\bar{T}_{\text {total }}}
\end{aligned}
$$

where $\overline{P E R_{S \rightarrow D}}=$ rate of packet error between the source and the destination, $\bar{P}_{\text {load }}$ a=average packet payload, $\bar{T}_{D}=$ average time for a single data packet transmission from the source to the destination, and $\bar{T}_{\text {total }}=$ total time required to complete a cooperative transmission through relays. The comparis on graph of the proposed TPC with the existing random linear coding (RLC), NCCARQ-MAC and Huffman coding in terms of throughput is depicted in Figure 4.

Figure 4 shows that the throughput of the proposed TPC is significantly higher than the existing RLC, NCCARQ-MAC and Huffman coding techniques. The throughput value of the proposed TPC is $5.42 \times 10^{8}(\mathrm{~b} / \mathrm{s})$, RLC is $4.53 \times 10^{8}(\mathrm{~b} / \mathrm{s})$ comparable to the Huffman coding, which achieves $4.09 \times 10^{8}(\mathrm{~b} / \mathrm{s})$. The NCCARQ-MAC achieves worst throughput value with $3.37 \mathrm{X} 10^{8}(\mathrm{~b} / \mathrm{s})$. However, Figure 3 shows that the TPC outperformed the existing NCCARQ-MAC, RLC and Huffman coding.

\subsection{Hop delay}

The total time from the transmission of data to its reception at the destinations is represented mathematically as:

$$
\bar{T}_{\text {time }}=\bar{T}_{D}+\bar{T}_{\text {co-operative }}
$$

where, $\bar{T}_{D}=$ average transmission time of one data packet from source to destination, $\bar{T}_{\text {co-operative }}=$ average time required to complete a cooperative transmission through relays. The average time spent at the cooperation phase is represented as: 


$$
\bar{T}_{\text {co-operative }}=\bar{T}_{\text {min-delay }}+\bar{T}_{\text {additional }}
$$

where, $\bar{T}_{\min -\text { delay }}=$ minimum average delay if there should be perfect scheduling among the relay, $\bar{T}_{\text {additional }}=$ additional delay due to the contention phase which has been received in our protocol for the probability of collisions to be decreased. The comparis on graph of proposed TPC with existing random linear coding (RLC), NCCARQ-MAC and Huffman coding in regards to hop delay is depicted in Figure 5.

Figure 5 shows that the hop delay of our proposed TPC hop delay is $9.72 \times 10^{-3}$ seconds which is significantly lesser than the existing RLC, NCCARQ-MAC and Huffman coding methods. The hop delay value of the existing RLC is 0.010 seconds, Huffman coding achieves 0.012 seconds. The NCCARQ-MAC results in the worst hop delay value with 0.012247 seconds. Regarding hop delay, the relays' dynamic nature has affected the networks' stability, especially in the NC scenario where it was difficult to predict the networks' behavior. However, for the TPC, the performance was better than the NCCARQ-MAC.

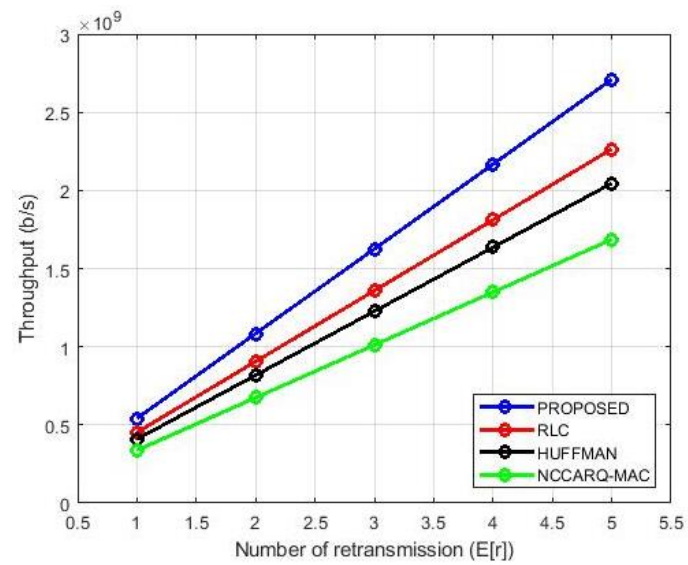

Figure 4. Comparison graph in terms of throughput

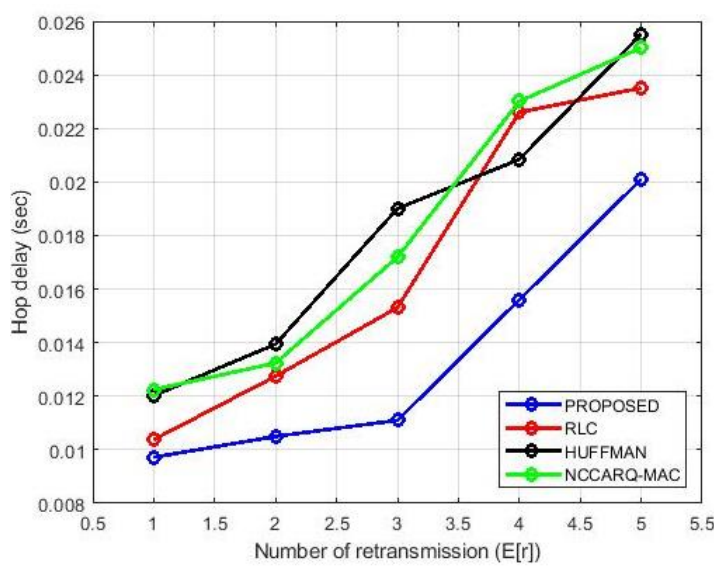

Figure 5. Comparis on graph in terms of hop delay

\subsection{Block error rate}

Block Error Rate is characterized as the fraction between numbers of errors packets to the total number of packets trans mitted at a certain time. The block error rate is denoted as,

$$
E_{\text {block }}=\frac{N_{\text {error }}}{N_{\text {total }}}
$$

Where, $E_{\text {block }}$ is the block error rate, $N_{\text {error }}$ is the number of packets has errors, $N_{\text {total }}$ is the total quantity of packets transferred at a specific time. The comparison graph of proposed TPC with existing random linear coding (RLC), NCCARQ-MAC and Huffman coding in regards of block error rate is depicted in Figure 6.

Figure 6 exhibits that the block error rate of our proposed TPC is significantly lower than the existing RLC, NCCARQ-MAC and Huffman coding techniques. The block error rate of the proposed TPC is 0.10 , and existing RLC is 0.15 , Huffman coding is 0.16 . The existing NCCARQ-MAC results in worst block error rate value with 0.20 .

\subsection{Packet delivery ratio}

This is the ratio of data packets received at the destinations to those transmitted from the source. Mathematically, it is denoted as:

$$
\overline{P D R}=\frac{\sum P_{\text {received }}}{\sum P_{\text {generated }}}
$$

Where, $\overline{P D R}=$ packet delivery ratio, $\sum P_{\text {received }}=$ sum of data packets gotten by every each destination, and $\sum P_{\text {generated }}=$ sum of data packets created by every source. The comparis on graph of proposed TPC with existing random linear coding (RLC), NCCARQ-MAC and Huffman coding regarding of packet delivery ratio is depicted in Figure 7. 
Figure 7 shows that the packet delivery ratio of our proposed TPC is significantly better than the existing RLC, NCCARQ-MAC and Huffman coding methods. The packet delivery ratio of the proposed TPC is $92.81 \%$ and the existing RLC is $90.62 \%$, Huffman coding achieves $88.42 \%$. The worst packet delivery ratio of $87.22 \%$ is attained for existing NCCARQ-MAC. The networks' stability during stationary relay results to increased packet delivery ratio by the developed TPC as depicted in Figure 7; however, the mobility and the frequent relay changes lowered the packet delivery ratio in the existing RLC, NCCARQ-MAC and Huffman coding as shown in Figure 7.

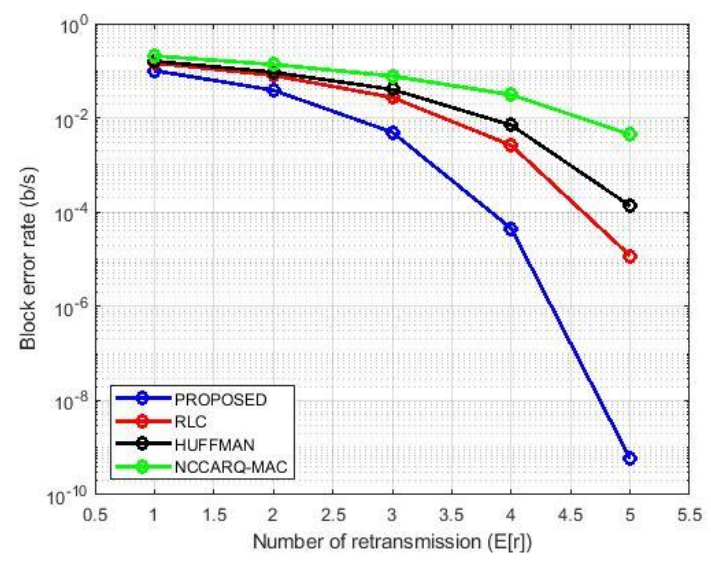

Figure 6. Comparison graph in terms of block error rate

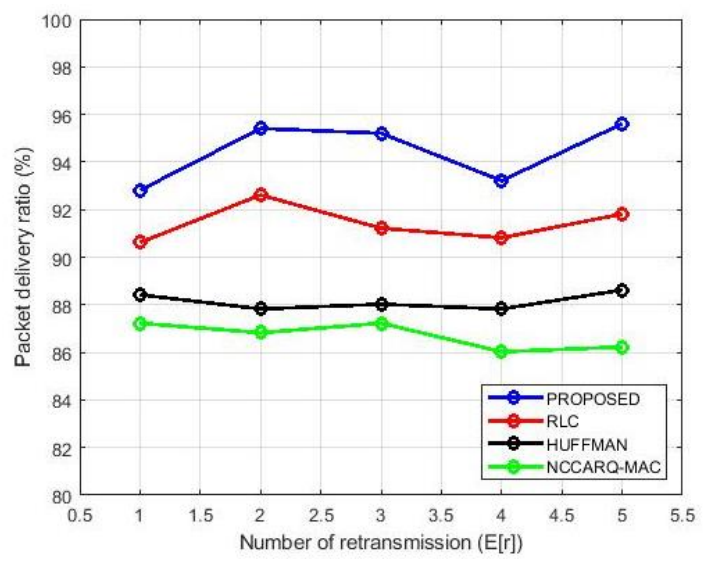

Figure 7. Comparis on graph in terms of packet delivery ratio

\subsection{Network decoder outage probability}

Consider the direct transmission of the source message $\left(\tilde{X}_{t}\right)$ to the destination. When the prompt limit of the channel over which the message is transferred becomes less than the desired rate $D_{r}($ bit $/ \mathrm{sec} / \mathrm{Hz})$, then the destination incorrectly decodes the transferred message, and an outage is said to happen. Let $\tilde{X}_{t}$ be the representation of $\tilde{X}_{t}$ when it is in outage. The outage probability is represented as:

$$
\begin{aligned}
& P\left(\tilde{X}_{t}\right)=P\left(\gamma_{s, d}<2^{\bar{R}}-1\right) \\
& =1-\exp \left(-\frac{2^{\bar{R}}-1}{\Gamma_{s, d}}\right)
\end{aligned}
$$

Where, $\Gamma_{s, d}=$ transmission path average SNR measured, $\bar{R}=$ exchanged replaced by $2^{\bar{R}}$ in repetition and AQLG network coding-based protocols, and by $\bar{R}$ in phases one and two respectively of coded cooperation protocol because of the shorter time available per phase. The comparison graph of proposed TPC with existing random linear coding (RLC), NCCARQ-MAC and Huffman coding in terms of network decoder outage probability is shown in Figure 8.

Figure 8 shows that the network decoder outage probability of our proposed TPC is significantly higher than the existing RLC, NCCARQ-MAC and Huffman coding techniques. The outage probability value of the proposed TPC is 0.81 , and the existing RLC outage probability is 0.975 , Huffman coding outage probability is 0.987 . Here, the existing NCCARQ-MAC provides the worst packet delivery ratio value with 1 and the proposed TPC provides the best packet delivery ratio value is 0.81 .

\subsection{Latency}

Latency is the time data packet takes to reach the destination. The formula utilized for calculating latency is given in (18).

$$
L_{y}=\frac{\sum\left(T_{\text {arrive }}-T_{\text {send }}\right)}{\sum N_{\text {linknodes }}}
$$


Where, $L_{y}=$ latency, $T_{\text {arrive }}=$ arrival time, $T_{\text {send }}=$ sending time, $N_{\text {linknodes }}=$ number of link nodes. The comparison graph of proposed TPC with existing random linear coding (RLC), NCCARQ-MAC and Huffman coding in terms of latency is shown in Figure 9.

Figure 9 exhibits that the latency of our proposed TPC is significantly lesser th an the existing RLC, NCCARQ-MAC and Huffman coding techniques. The latency value of the proposed TPC is $9.72 \times 10^{-3}$, existing NCCARQ-MAC is $1.52 \times 10^{-6}$ and Huffman coding is $1.59 \times 10^{-6}$. Here, the existing RLC provides the worst latency with $1.45 \times 10^{-6}$ seconds and the proposed TPC provides the best latency with $9.72 \times 10^{-3}$ seconds.

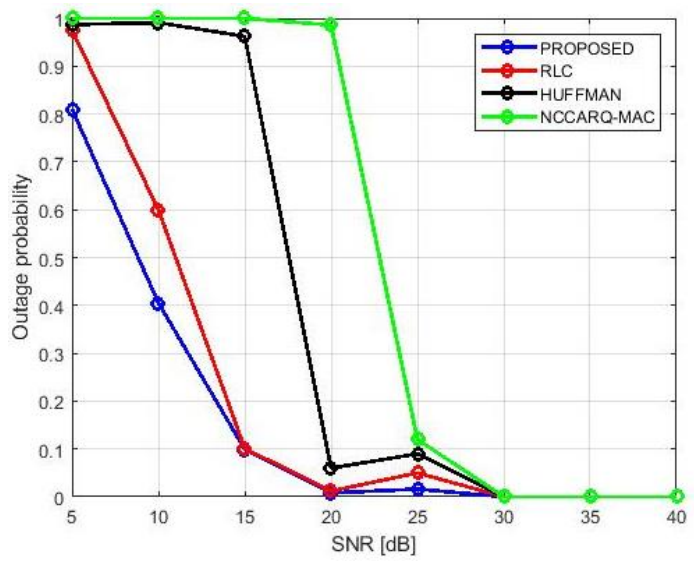

Figure 8. Comparison graph in terms of outage probability

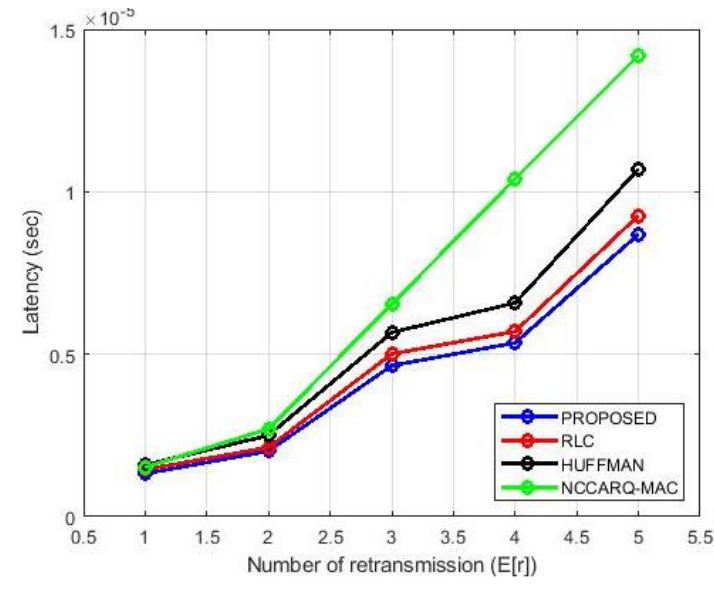

Figure 9. Comparison graph in terms of latency

\section{CONCLUSION}

This paper presented a transmit packet coding with the cuckoo search-based optimized relay nodes in VANETs. The presented coding technique supports the effective encoding of data packets from specific information flows to improve network performance. This work provides significant insights in support of the proposed TPC-based routing protocols in VANET. In the proposed technique, at first, nodes in the VANET are chosen using the trust- based cuckoo search optimization for secure data transmission. Moreover, the TPC secures the data in the transmission. The experimental outcomes showed that our proposed technique outperformed the existing techniques in terms of performance measures such as network delay, packet delivery ratio, block error rate, outage probability, latency, and throughput. In future work, improved optimization technique can be used for the effective relay selection, as well as develop the improved network coding.

\section{REFERENCES}

[1] T. Matsuda, T. Noguchi and T. Takine, "Survey of network coding and its applications," IEICE Transactions on Communications, vol 94, no. 3, pp. 698-717, 2011.

[2] O. A. Hammood, N. Nizam, M. Nafaa and W. A. Hammood, "RESP: Relay Suitability-based Routing Protocol for Video Streaming in Vehicular Ad Hoc Networks." International Journal of Computers, Communications \& Control, vol. 14, no. 1, pp.21-28, Feb 2019.

[3] A. S. Abdullah, M. J. Abbasi and N. Fisal, "Review of rateless-network-coding-based packet protection in wireless sensor networks" Mobile Information Systems, 2015.

[4] O. A. Hammood, M. N. M. Kahar, M. N. Mohammed, W. A. Hammood and J. Sulaiman, "The VANET-Solution Approach for Data Packet Forwarding Improvement," Advanced Science Letters 24, no. 10, pp. 7423-7427, 2018.

[5] S. Zeadally, R. Hunt, Y.-S. Chen, A. Irwin and A. Hassan, "Vehicular ad hoc networks (VANETS): Status, results, and challenges," Telecommunication Systems, vol. 50, no. 4, pp. 217-241, 2012.

[6] O. A. Hammood, M. N. M. Kahar and M. N. Mohammed, "Enhancement the video quality forwarding Using Receiver-Based Approach(URBA) in Vehicular Ad-Hoc Network," 2017 International Conference on Radar, Antenna, Microwave, Electronics, and Telecommunications (ICRAMET), Jakarta, 2017, pp.64-67.

[7] T. O. Fahad, A. A. Ali, "Compressed fuzzy logic based multi-criteria AODV routing in VANET environment" International Journal of Electrical and Computer Engineering, vol. 9, no. 1, pp. 397-401, 2019

[8] S. Al-Sultan, M. M.Al-Doori, A. H. Al-Bayatti and H. Zedan, "A comprehensive survey on vehicular ad hoc network" Journal of network and computer applications, vol. 37, pp. 380-392, 2014. 
[9] S. P. Godse and P. N. Mahalle, "A Computational Analysis of ECC Based Novel Authentication Scheme in VANET," International Journal of Electrical and Computer Engineering, vol. 8, no. 6, pp. 5268-5277, 2018.

[10] M. Li, Z. Yang and W. Lou, "CodeOn: Cooperative Popular Content Distribution for Vehicular Networks using Symbol Level Network Coding," in IEEE Journal on Selected Areas in Communications, vol. 29, no. 1, pp. 223-235, January 2011.

[11] A. Ayoob, G. Su, D. Wang, M. Mohammed and O. A. Hammood. "Hy brid LTE-VANETs based optimal radio access selection." In International Conference of Reliable Information and Communication Technology, pp. 189-200. Springer, Cham, 2017.

[12] S. Sharma, D. Jindal and R. Agarwal, "Analysing Mobile Random Early Detection for Congestion Control in Mobile Ad-hoc Network, International Journal of Electrical and Computer Engineering, vol. 8, no. 3, pp. 1305-1314, 2018.

[13] S. Xu, P. Guo, B. Xu and H. Zhou, "QoS evaluation of VANET routing protocols." Journal of Networks, vol 8, no. 1, pp. 132-139, 2013.

[14] S.A.M. Ahmed, S. H. S. Ariffin and N. Fisal. "Network coding techniques for VANET advertising applications" EURASIP Journal on Wireless Communications and Networking, 2015.

[15] C. Wu, X. Chen, Y. Ji, S. Ohzahata and T. Kato, "Efficient Broadcasting in VANETs Using Dynamic Backbone and Network Coding," IEEE Transactions on Wireless Communications, vol.14, no.11, pp.6057-6071, Nov. 2015.

[16] R. A. Hasan, M. N. Mohammed, M. A. Bin Ameedeen and E. T. Khalaf, "Dynamic Load Balancing Model Based on Server Status (DLBS) for Green Computing." Advanced Science Letters, vol. 24, no. 10, pp. 7777-7782, 2018.

[17] M. A. Elfattah, A. E. Hassanien and S. Abuelenin, "A hybrid swarm optimization approach for document binarization," Stud. Informat. Control, vol. 28, no. 1, pp. 65-76, Mar 2019.

[18] C. Suthaputchakun, M. Dianati and Z. Sun, "Trinary Partitioned Black-Burst-Based Broadcast Protocol for TimeCritical Emergency Message Dissemination in VANETs," in IEEE Transactions on Vehicular Technology, vol. 63, no. 6, pp. 2926-2940, July 2014.

[19] C. Wu, S. Ohzahata and T. Kato, "VANET broadcast protocol based on fuzzy logic and lightweight retransmission mechanism," IEICE transactions on communication, vol. E95, no. 2, pp.415-425, 2012

[20] R. S. Jadhav and M. M. Dongre, "Performance enhancement of vanets using fuzzy logic and network coding," International journal of advanced computational engineering and networking, vol. 5, no. 2, pp. 39-42, Feb 2017.

[21] Y. Yang, W. Chen, O. Li, Q. Liu and L. Hanzo, "Truncated-ARQ Aided Adaptive Network Coding for Cooperative Two-Way Relaying Networks: Cross-Layer Design and Analy sis," in IEEE Access, vol. 4, pp. 9361-9376, 2016.

[22] H. Chiu and S. Wu, "Cross-Layer Performance Analysis of Cooperative ARQ With Opportunistic Multi-Point Relaying in Mobile Networks," in IEEE Transactions on Wireless Communications, vol. 17, no. 6, pp. 4191-4205, June 2018

[23] A. Roy and R. Roy, "Reliability benefit of network coding and cooperative communication," Physical Communication, vol. 29, pp. 217-229, Aug 2018.

[24] L. Li, Z. Yang, J. Wang, Z. S. Kui and Y. Zhu "Network coding with crowdsourcing-based trajectory estimation for vehicular networks," Journal of Network and Computer Applications, vol. 64, pp. 204-215, 2016.

[25] A. Antonopoulos, A. S. Lalos, M. Di Renzo and C. Verikoukis, "Cross-Layer Theoretical Analysis of NC-Aided Cooperative ARQ Protocols in Correlated Shadowed Environments," in IEEE Transactions on Vehicular Technology, vol. 64, no. 9, pp.4074-4087, Sept. 2015.

[26] W. Libing, F. Jing, W. Jing, N. Lei and W. Hao. "Emergency Message Broadcast Method Based on Huffinan-Like Coding," Journal of Computer Research and Development, vol. 54, no. 11, pp. 2475-2486, 2017.

[27] F. Liu, Z. Chen and B. Xia, "Data Dissemination With Network Coding in Two-Way Vehicle-to-Vehicle Networks," in IEEE Transactions on Vehicular Technology, vol. 65, no. 4, pp. 2445-2456, April 2016.

[28] N. M. A. Brahin, H. M. Nasir, A. Z. Jidin, M. F. Zulkifli and T. Sutikno, "Development of Vocabulary Learning Application by Using Machine Learning Technique," Bulletin of Electrical Engineering and Informatics, vol. 9, no.1, pp. 362-369, 2020.

[29] M. Mabrok, Z. Zakaria, T. Sutikno and A. Alhegazi, "Wideband power amplifier based on Wilkinson power divider for s-band satellite communications," Bulletin of Electrical Engineering and Informatics, vol. 8, pp. 1531-1536, 2019.

[30] R. A. Hasan, M. A. Mohammed, Z. H. Salih, M. A. B. Ameedeen, N. Țăpuş and M. N. Mohammed, "HSO: A Hybrid Swarm Optimization Algorithm for Reducing Energy Consumption in the Cloudlets," Telecommunication, Computing, Electronics and Control, vol. 16, no. 5, pp. 2144-2154, 2018.

[31] M. A. Ahmed, R. A. Hasan, A. H. Ali and M. A. Mohammed, "The classification of the modern arabic poetry using machine learning," Telecommunication, Computing, Electronics and Control, vol. 17, no. 5, pp. 2667-2674, 2019.

[32] M. A. Mohammed, R. A. Hasan, M. A. Ahmed, N. Tapus, M. A. Shanan, M. K. Khaleel, et al., "A Focal load balancer-based algorithm for task assignment in cloud environment," in 2018 10th International Conference on Electronics, Computers and Artificial Intelligence (ECAI), 2018, pp.1-4.

[33] M. A. Mohammed and N. ȚĂPUŞ, "A novel approach of reducing energy consumption by utilizing enthalpy in mobile cloud computing," Studies in Informatics and Control, vol. 26, pp. 425-434, 2017.

[34] Hasan, R. A. and M. N. Mohammed, "A krill herd behaviour inspired load balancing of tasks in cloud computing," Studies in Informatics and Control, vol. 26, no. 4, pp.413-424, 2017.

[35] N. Q. Mohammed, M. S. Ahmed, M. A. Mohammed, O. A. Hammood, H. A. N. Alshara and A. A. Kamil, "Comparative Analysis between Solar and Wind Turbine Energy Sources in IoT Based on Economical and Efficiency Considerations," 2019 22nd International Conference on Control Systems and Computer Science (CSCS), Bucharest, Romania, 2019, pp. 448-452. 